\title{
Erratum: The Stress Tensor for the Mixed Phase of Uniaxial Anisotropic Superconductors*
}

\author{
V. G. Kogan \\ Technion-Israel Institute of Technology, Haifa, Israel
}

(Received April 17, 1979)

To the list of references should be added:

10. Ian Easson and C. J. Pethick, Phys. Rev. D 16, 275 (1977).

${ }^{*}$ This paper appeared in $J$. Low Temp. Phys. 34(3/4), 335 (1979). 